\title{
A rare but life-threatening pneumopathy induced by Olaparib: From a clinical case to a review of literature
}

\author{
Grisay G, Percy C, Pierrard J and Seront E* \\ Department of Medical Oncology, Centre Hospitalier de Jolimont, Rue Ferrer 159, 7100 Haine Saint Paul, Belgium
}

\begin{abstract}
The diagnosis of drug-induced pneumopathy remains a challenge for clinicians, particularly in oncology field in which many new drugs are more and more used and induce uncommon adverse events by targeting specific pathways. We report the case of a patient with ovarian cancer who developed a rapidly progressive and lifethreatening pneumopathy after initiation of olaparib. High dose of corticosteroids rapidly (in 3 days) improved the symptoms, decreased the oxygen supply and led to normalization of lung imaging. Olaparib-induced pneumopathy is rarely reported in literature. We discuss about the role of Poly (ADP-Ribose) polymerase (PARP) enzyme in the lung homeostasis and highlight the importance of consider high-doses steroids when suspecting this specific drug-induced pneumopathy.
\end{abstract}

\section{Introduction}

Drug-induced pneumopathy remains a diagnosis challenge for clinicians in oncology field. Indeed, new drugs are more and more used in patients who are sometimes frail and previously heavily treated. Furthermore, these agents are used sequentially or concomitant with other combinations, reflecting the difficulty to identify the real causative agent. All these drugs target different signaling pathways and can therefore deregulate homeostasis, resulting sometimes in unexpected adverse events. We report the case of a patient who developed a rapidly progressive pneumopathy after initiation of olaparib. Although rarely associated with pulmonary symptoms, olaparib appeared as the first causative agent in this case; we described the diagnostic and therapeutic strategy and we review the influence that olaparib could have on the lung homeostasis.

\section{Case-report}

A 71-year woman with ovarian cancer was admitted for rapidly progressive dypnea. Her non-oncologic medical history was not relevant; she never smoked and was regularly vaccinated against flu and pneumonia. In 2014, she was diagnosed with an ovarian adenocarcinoma involving the peritoneal cavity and classified as Fédération Internationale de Gynécologie et d'Obstétrique (FIGO) III. Chemotherapy with carboplatin-paclitaxel (6 cycles, every 3 weeks) resulted in radiological complete response (following RECIST criteria version 1.1) and in normalization of tumor marker CA-125. Debulking surgery was then performed and removed all macroscopically tumor lesions. Germline and tumoral analysis did not demonstrate any DNA repair mechanisms defect, such as $B R C A-1$ or 2 deficiencies. In October 2016, recurrence of cancer was confirmed with apparition of liver metastases (proven by biopsy), peritoneal carcinomatosis and CA-125 increase. Six cycles of carboplatin-gemcitabine in association with the anti-Vascular Endothelial Growth Factor bevacizumab induced complete radiological response and CA-125 normalization. Bevacizumab was then continued till radiological progression in October 2017. Chemotherapy associating liposomal doxorubicine and trabectidine allowed a stabilization of the disease till cancer progression observed in August 2018 (apparition of new liver metastases and increase of CA-125 to $1.200 \mathrm{kU} / \mathrm{L}$ ). Carboplatine-gemcitabine regimen (6 cycles, one cycle every 3 weeks) was thus started and resulted in January 2019 in radiological partial response and an important decrease of CA-125 (30 kU/L). The patient was completely asymptomatic and the thoracic CT was completely normal. Olaparib, an inhibitor of the Poly (ADP-Ribose) Polymerase (PARP), was then started in maintenance at a daily dose of $300 \mathrm{mg}$ twice a day.

Six weeks after olaparib initiation, she was admitted for severe dyspnea appearing at rest. She described a 2-week history of breath shortness that rapidly progressed, without any temperature, chills or cough. Clinical examination revealed bilateral fine inspiratory crackles. Arterial blood gas analysis revealed hypoxemia $(\mathrm{O} 2=52$ $\mathrm{mmHg})$ and hypocapnia $(\mathrm{CO} 2=24 \mathrm{mmHg})$. Thoracic CT excluded pulmonary embolism but demonstrated bilateral lung infiltrates with ground glass opacities (Figure 1A). C-reactive protein (CRP) was moderately increased $(65 \mathrm{mg} / \mathrm{dl}$ for normal range between $0-20 \mathrm{mg} /$ $\mathrm{dL}$ ). The rest of biology was within normal range, including CA-125 (25 kU/L). Medications include aspirin $80 \mathrm{mg}$ daily and ocasionnal use of paracetamol for knee arthrosis. Olaparib was stopped and intravenous antibiotherapy (piperacillin/tazobactam) associated with clarithromycin was started. Due to the absence of clinical improvement and biological improvement after 5 days, a broncho-alveolar lavage (BAL) was performed and showed abundance of lymphocytes T-cells with a normal ratio helper/suppressor $(C D 4 / C D 8=0.96)$. There was no eosinophil and a low quantity of macrophages. Culture remained sterile. All bacteriological cultures remained sterile; serologies for mycoplasma, chlamydia, aspergillus, pneumocystis, Epstein Barr Virus and cytomegalovirus were negative; polymerase chain reaction for

*Correspondence to: Emmanuel Seront, Department of Medical Oncology, Centre Hospitalier de Jolimont Rue Ferrer 159, 7100 Haine Saint Paul, Belgium, E-mail: emmanuel.seront@jolimont.be

Key words: Olaparib, pneumopathy, adverse event, corticosteroids, PARP

Received: December 10, 2019; Accepted: December 23, 2019; Published: December 26, 2019 
legionella, chlamydia and mycoplasma pneumoniae were all negative, as well as the antigen for influenza and the urine antigen for legionella. Auto-immune serologies (antinuclear factor and rheumatoid factor) were negative. Cardiac ultrasonography showed a normal ejection fraction. Lymphangitis carcinomatosis was not suspected as CA-125 was normal and as no malignant cell was isolated in the BAL. Due to the frailty of our patient and the increasing oxygen supply, we were unable to perform a lung biopsy.

After exclusion of infectious cause, we started intravenous bolus of high-dose corticosteroids (methylprednisone $500 \mathrm{mg}, 3$ times daily). Patient experienced a rapid respiratory improvement after only 3 days, with a progressive decrease in oxygen administration. On day 4, methylprednisone was thus decreased to $125 \mathrm{mg}$ daily for 3 days and then tapered slowly. The CT scan performed 7 days after steroids initiation showed a decrease of lung infiltrates (Figure 1B) and a complete resolution after only 8 weeks (Figure 1C). Olaparib was not reintroduced. Four months later, thoracic $\mathrm{CT}$ was normal but abdominal CT showed majoration of liver metastases, peritoneal carcinomatosis and CA-125 increase (14.000 kU/L). New chemotherapy (oxaliplatinbased regimen) was started but she died from liver insufficiency due to cancer progression three months later. The last thoracic CT, performed before her death, did not show any lung anomalies.

\section{Discussion}

We describe a case of life-threatening pneumopathy occurring after olaparib instauration for ovarian cancer. The PARP inhibitor olaparib was started in our patient despite the absence of germline or somatic BRCA mutation. Although originally approved for BRCAmutated ovarian cancer patients only, the long-term efficacy observed from different trials, including the Study 19 and the phase III SOLO2 trial in both $B R C A$-mutated and non $B R C A$-mutated patients led to expanding the use of olaparib in the maintenance treatment of patients with recurrent epithelial ovarian, fallopian tube or primary peritoneal cancer who are in a complete or partial response to platinum-based chemotherapy, irrespective of their $B R C A$ mutated status $[1,2]$.

In clinical trials, olaparib was rarely associated with pulmonary symptoms, dyspnea appearing in less than $1 \%$ of patients [1,2]. PARPs constitute an 18-member family of proteins that play an important role in DNA repair. PARP-1 is the most abundant and studied member of this family. It is mostly located in cell nuclei and is strongly activated by DNA strand breaks due to reactive oxygen species Although the primary aim of PARP-1 is to maintain the genome integrity, PARP1 can also promote inflammation by regulating the expression of several NF- $\mathrm{KB}$-dependent cytokines, chemokines, adhesion molecules, inducible nitric-oxide synthase [3]. PARP-1 is involved in a number of fibrotic and inflammatory disorders affecting heart, liver, vessels and lung [4-9]. In lung, genetic depletion and pharmacological inhibition of PARP-1 were shown to reduce pulmonary fibrosis in an animal model of bleomycin-induced lung injury, suggesting that PARylation

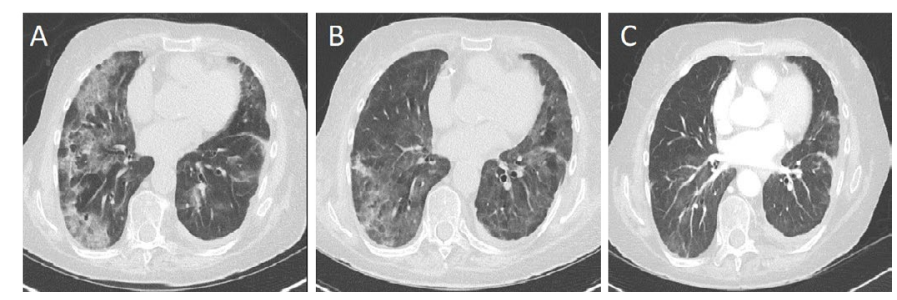

Figure 1. Thoracic Computed Tomography evaluation (A) 4 weeks after olaparib initiation; (B) 7 days after corticoids initiation; (C) 2 month after corticoids initiation is important for myofibroblast differentiation and for the pathogenesis of the disease [8,9]. PARP-1 inhibitors were also shown to reduce the eosinophil recruitment in asthmatic lungs, confirming the role of PARP-1 in asthma pathogenesis [10]. Conversely, other studies reported opposite results; PARP deficiency has also been associated with excessive lung remodeling and inflammation after lung injury, through high expression of inflammatory cytokines such as IL-6, CXCL13 or IGFBP-5, which have been shown to be expressed in idiopathic pulmonary fibrosis lung tissues $[11,12]$. These controversial role of PARP-1 suggest that the role of this enzyme in lung homeostasis remains unclear.

In our patient, we highly suspect olaparib to play a role in the development of the pneumopathy, due to the close relationship with olaparib initiation, the normal thoracic CT before olaparib initiation and the absence of other cause. Bacteriology (in blood and BAL) remained sterile and antibiotherapy did not improve the evolution suggesting a non-infectious cause; the normal CA-125 on olaparib, the absence of malignant cells isolated in $\mathrm{BAL}$ and the rapid regression of lung infiltrates exclude cancer evolution; no other medication has been involved and there was no sign of auto-immune disease. The rapid regression on corticosteroids suggest also a high inflammatory process, which could be consistent with in vivo models $[11,12]$.

Other anti-cancer medications have been associated with pneumopathy, including cytotoxic agents (docetaxel, paclitaxel, bleomycine or gemcitabine), targeted therapies (everolimus, temsirolimus, cetuximab) or immune checkpoint inhibitors (nivolumab, atezolizumab, pembrolizumab, ipilimumab); however, incidence is rare and the drug-induced pulmonary toxicity remains a diagnostic of exclusion $[13,14]$. Clinical presentations may vary from cough to acute respiratory distress syndrome. Timing of clinical manifestation between the start of the therapy and the onset of symptoms is also unpredictable. Radiological findings are also inconsistent and may vary from mild isolated pulmonary opacities to multiple lobar consolidations. Workup should include thoracic CT, BAL and lung biopsy. The BAL is mandatory in order to rule out an infection or an alveolar haemorrhage. It also can provide essential information regarding the cell counts as it may show neutrophilia, lymphocytosis or rarely eosinophilia. More relevant is the ratio $\mathrm{CD} 4 / \mathrm{CD} 8$. Usually, the ratio is lowered in a drug-induced pneumonitis, but a normal ratio is also observed in drug-induced pneumopathy, like in our patient case $[13,14]$. Recently, Terbuch et al. evaluated clinical and radiological parameters influencing druginduced pneumopathy in cancer patients participating in phase I clinical trials. They showed that the number of affected lobes and steroid therapy did not influence an improvement while continuation of the causal agent resulted in worsening of the outcome [15]. Interestingly, in our patient, improvement occurred rapidly (in 3 days) after high-dose steroids initiation, suggesting a benefit of such treatment in olaparibinduced pneumopathy, which maybe differ from other pneumopathy induced by different antitumoral agents.

Of course, we cannot certify that olaparib is the cause of this pneumopathy and other causes could have facilitated the development of this disease, such as the previous therapies our patient received or other unknown inflammatory aetiologies. We cannot confirm the role of steroids in regression of infiltrates, even if there was a close relationship between the steroid's onset and the improvement of our patient. Finally, we do not know whether a biopsy of the lung could had identified the real cause. 


\section{Conclusion}

In conclusion, drug-induced pneumopathy should always be suspected in patient developing dyspnea, particularly when a new drug has recently been initiated, in absence of clear etiology and absence of improvement with antibiotherapy. In this case, olaparib could appear as a cause of pneumopathy. Rapid identification of this cause may allow its rapid interruption and high dose of corticosteroids should be considered in case of life-threatening situations.

\section{References}

1. Friedlander M, Matulonis U, Gourley C (2018) Long-term efficacy, tolerability and overall survival in patients with platinum-sensitive, recurrent high-grade serous ovarian cancer treated with maintenance olaparib capsules following response to chemotherapy. Br J Cancer 119: 1075-1085.

2. Pujade-Lauraine E, Ledermann JA, Selle F (2017) Olaparib tablets as maintenance therapy in patients with platinum-sensitive, relapsed ovarian cancer and a BRCA1/2 mutation (SOLO2/ENGOT-Ov21): a double-blind, randomised, placebocontrolled, phase 3 trial. Lancet Oncol 18: 1274-1284.

3. Pommier Y, O'Connor MJ, de Bono J (2016) Laying a trap to kill cancer cells: PARP inhibitors and their mechanisms of action. Sci Transl Med 8: 362

4. Pacher P, Liaudet L, Bai P (2002) Activation of poly (ADP-ribose) polymerase contributes to development of doxorubicin-induced heart failure. J Pharmacol Exp Ther 300: 862-867.

5. Gero D, Szoleczky P, Chatzianastasiou A (2014) Modulation of poly (ADP-ribose) polymerase-1 (PARP-1)-mediated oxidative cell injury by ring finger protein 146 (RNF146) in cardiac myocytes. Mol Med 20: 313-328.
6. Mukhopadhyay P, Rajesh M, Cao Z (2014) Poly (ADP-ribose) polymerase-1 is a key mediator of liver inflammation and fibrosis. Hepatology 59: 1998-2009.

7. Meloche J, Pflieger A, Vaillancourt M, et al (2014) Role for DNA damage signaling in pulmonary arterial hypertension. Circulation 129: 786-797.

8. Genovese T, Mazzon E, Di Paola R (2005) Inhibitors of poly (ADP-ribose) polymerase modulate signal transduction pathways and the development of bleomycin-induced lung injury. J Pharmacol Exp Ther 313: 529-538.

9. Lucarini L, Durante M, Lanzi C (2017). HYDAMTIQ, a selective PARP-1 inhibitor, improves bleomycin-induced lung fibrosis by dampening the TGF- $\beta$ /SMAD signalling pathway. J Cell Mol Med 21: 324-335

10. Lucarini L, Pini A, Gerace E (2014) Poly (ADP-ribose) polymerase inhibition with HYDAMTIQ reduces allergen-induced asthma-like reaction, bronchial hyper-reactivity and airway remodelling. J Cell Mol Med 18: 468-479.

11. Ke Y, Zhang J, Lv X (2019) Novel insights into PARPs in gene expression: regulation of RNA metabolism. Cell Mol Life Sci 76: 3283-3299.

12. Su Y, Nishimoto T, Feghali-Bostwick C (2015) IGFBP-5 promotes fibrosis independently of its translocation to the nucleus and its interaction with nucleolin and IGF. PLoS One 10: e 0130546.

13. Vahid B, Marik PE (2008) Pulmonary complications of novel antineoplastic agents for solid tumors. Chest 133: 528-538.

14. Yonemori K, Hirakawa A, Kawachi A (2016) Drug induced interstitial lung disease in oncology phase I trials. Cancer Sci 107: 1830-1836.

15. Terbuch A, Moreno Candilejo I, Scaranti M (2019) Distinct radiological patterns of drug-induced pneumonitis (R-DIP) in early-phase clinical trials and predictive factors affecting outcome: A 10-year systematic review from the Royal Marsden hospital phase i drug development unit experience. J Clin Oncol 37: 3088-3088.

Copyright: (C2019 Grisay G. This is an open-access article distributed under the terms of the Creative Commons Attribution License, which permits unrestricted use, distribution, and reproduction in any medium, provided the original author and source are credited. 